\title{
Control of genital chlamydial infection
}

Paul R Gully MB ChB FrCPC ${ }^{1}$, ROSANNA W PeELING PhD ${ }^{2}$

S EXUALLY TRANSMITTED DISEASES (STD) REMAIN THE MOST common reportable infections in Canada even though there have been encouraging trends of decreasing incidence of some STD. Rates of reporting of gonorrhea are now below 50/100,000 for males and females, and rates of reporting of 'infectious syphilis' are very low, following increases in 1990 and 1991. Rates of reporting of hepatitis B remain around 10/100,000 but vary greatly by province and territory. The control of all STD involves: primary prevention, eg, sexual health education and promotion of risk reduction; secondary prevention, ie, screening for subclinical disease and treatment of contacts; and tertiary prevention, ie, treatment of clinical disease to prevent complications of disease. We are fortunate in Canada to have the resources to be able to monitor antimicrobial-resistant gonococcal infections and to use appropriate treatment. The fall in rates of 'infectious syphilis', which were increasing in young adults, predominantly females, may be a reflection of good public health practice. The tool is also at hand to control hepatitis B. Universal immunization has been introduced successfully in British Columbia, and other jurisdictions will probably follow. On the other hand, viral STD such as human papilloma virus (HPV) infection and genital herpes are highly prevalent among the young, and effective control strategies for primary prevention have yet to be identified. Recent technological advances in the diagnosis of cervical HPV infection enabling accurate identification of HPV types will require serious consideration in the formulation of guidelines for the management of women with abnormal Papanicolaou smear results.

It is the control of genital chlamydial infections that

\footnotetext{
${ }^{I}$ Division of Sexually Transmitted Disease Control, Bureau of Communicable Disease Epidemiology, ${ }^{2}$ National Laboratory for Sexually Transmitted Disease, Bureau of Microbiology. Laboratory Centre for Disease Control, Ottawa, Ontario Correspondence: Dr Paul R Gully. Chief. Division of Sexually Transmitted Disease Control, Bureau of Communicable Disease Epidemiology. Laboratory Centre for Disease Control, Tunney's Pasture, Ottawa, Ontario K1A OL2
}

presents one of the next major public health challenges. Genital chlamydia rates are showing a gradual decline in most jurisdictions but remain unacceptably high, especially in adolescents and young adults (1, unpublished data). The much higher rates in young females than in males is a reflection of testing and detection, and is probably not a real difference in epidemiology. The long term consequences of chlamydial infection are well described. The final report of the Royal Commission on New Reproductive Technologies recognized STD, especially chlamydia, to be the single most important preventable cause of infertility in Canada (2).

Chlamydial infections should be amenable to traditional measures of STD control because accurate diagnostic tests are widely available, and curative therapy is inexpensive and reasonably well tolerated. However, there are problems unique to the control of chlamydial infection: a large reservoir of asymptomatic infection; a long duration of infectiousness; and poor compliance with a seven-day course of antimicrobial therapy, partly because symptoms (when present) may resolve after three to four days. Targeted screening of those likely to comprise the reservoir of subclinical infection, eg, sexually active adolescents and young adults, has been recommended in STD guidelines (3) directed to primary care providers. The drawback has been that nonculture antigen detection type diagnostic tests, which have reasonable performance characteristics in symptomatic individuals, lack the sensitivity required for a population-based screening test (4), this being especially true in asymptomatic men. The use of enzyme linked immunoassay tests on first void urine specimens was intended to increase the ease of testing and screening men, but as yet, the sensitivity of these tests has been variable. Field evaluations have shown sensitivities ranging from 38 to $100 \%$ and specificities from 86 to $100 \%(5-9)$.

The recent introduction of molecular amplification techniques for the detection of Chlamydia trachomatis, such as polymerase chain reaction and ligase chain 
reaction assays, will probably result in a substantial improvement in the laboratory diagnosis of genital infections. As diagnostic and screening tests, they have been shown to offer performance characteristics equivalent to culture but are more rapid and have no special transport requirements $(10,11)$. These techniques also have the advantage of offering 'multiplex' assays when more than one pathogen that produces similar clinical signs and symptoms, eg, $C$ trachomatis or Neisseria gonorrhoeae, can be detected using a single specimen. However, inherent in the exquisite sensitivity of these amplification techniques is the potential for false positive results due to contamination. Crosscontamination between specimens, especially after amplification, and run-to-run contamination due to contamination of equipment, reagents and supplies, create problems in specimen handling and processing in a routine diagnostic laboratory. Field evaluations have also shown that some amplification reactions may be susceptible to inhibitory substances in the specimen, such as heme (from a bloody specimen), heparin and phosphate ions. Efforts to adapt these new technologies for routine use are ongoing. For the present, because of the psychosocial and medicolegal implications of a positive test for STD, a confirmatory assay for chlamydial infection is strongly recommended.

The third issue noted above is compliance with therapy. There is now more evidence of the effectiveness of quinolones, eg, ofloxacin (12), in the treatment of genital chlamydia. When the Canadian STD Guidelines (4) are reviewed in 1994/95, the position of the quinolones will no doubt be reconsidered. However, multiple doses are still required. It seems likely that the azalide, azithromycin (13), will be available in Canada in 1994. Azithromycin has the advantage of only requiring a single oral dose of $1 \mathrm{~g}$ for treatment due to its tissue retention time of five to seven days. This is important in the treatment of an intracellular organism such as $C$ trachomatis when other drugs have only good therapeutic ratios measured in terms of hours. There is good evidence from several clinical trials that single-dose azithromycin is equivalent to a seven-day regimen of tetracyclines (14-16). However, azithromycin is not highly efficacious against $N$ gonorrhoeae at the same dosage, and therefore two-drug therapy against combined infections would still be necessary. The effectiveness of azithromycin in cases of urethritis not caused by $N$ gonorrhoeae or $C$ trachomatis also has to be taken into account. Recent studies have not examined efficacy against specific organisms and only evaluate on the basis of a change in polymorphonuclear cell counts on Gramstained smears $(17,18)$.

At first sight the availability of a single-dose treatment for chlamydia seems to present the possibility of much greater success in chlamydial control, especially when compliance with tetracyclines and macrolides is demonstrably poor. However, we should be cautious.
Katz et al (19) showed a compliance rate of $63.4 \%$ in STD clinic clients treated for chlamydial infection, but Grob (20) cautions that compliance is dependent on four factors: the patient, the physician, the severity of the disease, and the frequency and duration of therapy. Physicians may be able to do more in counselling patients to encourage compliance, especially by emphasizing the long term consequences of disease. Whatever the costs of tetracyclines to governments and individuals, azithromycin will inevitably be more expensive initially. Comparison of costs with effectiveness has to take into account many factors, including compliance, requirements for retreatment, further contact tracing, transmission of infection from partially treated persons and long term effects of partially treated disease. If the rate of reporting of chlamydial infections continues to fall as a result of current control methods, then it is likely that newer, more expensive therapies should perhaps be considered as a second-line choice, except in situations where compliance is a major issue and where the extent of risky sexual behaviour is such that the aggressive use of a single-dose therapy for cases and contacts is justified. Therefore, the rate of infection and the circumstances of infection may have to be taken into account. Although structurally related to the macrolides, eg, erythromycin, azithromycin will likely be contraindicated in pregnancy due to lack of toxicological data. This will have to be taken into account in populations at high risk where the possibility of pregnancy may be increased.

Genç et al (21) carried out an economic evaluation of screening for $C$ trachomatis in adolescent males and concluded that, in combination with a single dose of azithromycin, urine screening using an enzyme immunoassay with or without leukocyte esterase testing improved cure rates and reduced costs. They note that the use of polymerase chain testing of urine would be less attractive due to laboratory contamination issues and cost. This study reflects the dilemma that public health practitioners are facing today. For the first time, we have very sensitive molecular techniques for screening and a single-dose drug that can help us overcome major problems in chlamydia control, but these innovations will cost five to 10 times more than conventional methods of diagnosis and therapy. These advances will, in our opinion, ultimately result in significantly reduced health care costs as a result of a reduction in expensive long term sequelae. We need to debate whether we can afford not to use these aids to chlamydia control and in what circumstances they can be used most cost-effectively.

\section{REFERENCES}

1. Gully PR, Rwetsiba D. Chlamydial infection in Canada. Can Dis Wkly Rep 1991;17:282-91.

2. Royal Commission on New Reproductive Technologies. Proceed with Care: Final Report of the Royal Commission on New Reproductive Technologies. Ottawa: Minister of Government Services Canada, 1993:199. 
3. Canadian guidelines for the prevention, diagnosis, management and treatment of sexually transmitted diseases in neonates, children, adolescents and adults 1992. Can Commun Dis Rep 1992;18(Suppl 1).

4. Albritton WL. The sexually transmitted diseases laboratory in a framework of population-based diagnostics. Sex Transm Dis 1992;19:279-83.

5. Hay PE. Thomas BJ, McKenzie P, Taylor-Robinson D. Detection of Chlamydia trachomatis in men: sensitive tests for sensitive urethras. Sex Transm Dis 1993;20:1-4.

6. Paul ID, Caul EO. Evaluation of three Chlamydia trachomatis immunoassays with an unbiased non-invasive clinical sample. J Clin Microbiol 1990;28:220-2.

7. Chernesky M, Castriano S, Senors J, et al. Detection of Chlamydia trachomatis antigens in urine as an alternative to swabs and cultures. J Infect Dis 1990;161:124-6.

8. Schwebke JR, Clark AM, Pettinger MB, Nsubga P, Stamm WE. Use of a urine enzyme immunoassay as a diagnostic tool for Chlamydia trachomatis urethritis in men. J Clin Microbiol 1991;29:2446-9.

9. Jensen IP. A comparison of urine sample to urethral swab for detection of Chlamydia trachomatis in asymptomatic young men using two enzyme immunoassays. Sex Transm Dis 1992;19:165-9.

10. Jaschek G, Gaydos CA, Welsh LE, Quinn TC. Direct detection of Chlamydia trachomatis in urine specimens from symptomatic and asymptomatic men using a rapid polymerase chain reaction assay. J Clin Microbiol 1993;31:1209-12.

11. Peeling R, Brunham RC. Molecular techniques for the laboratory identification of Chlamydia trachomatis. J lnt Fed Clin Chem. (In press)

12. Hooton TM, Battelger BE, Judson FN, Spraunce SL,
Stamm WE. Ofloxacin versus doxycycline for treatment of cervical infection with Chlamydia trachomatis. Antimicrob Agents Chemother 1992;36:1144-6.

13. Gold R. Beyond erythromycin. Can J Infect Dis 1994;5:13-4

14. Martin DH, Mroczkowski TF, Dalu ZA, et al. A controlled trial of single dose of azithromycin for the treatment of chlamydial urethritis and cervicitis. N Engl J Med 1992;327:921-5.

15. Steingrimsson O, Olafsson JH, Thorarinsson $\mathrm{H}$, et al. Single dose azithromycin treatment of gonorrhea and infections caused by $C$ trachomatis and $U$ urealyticum in men. Sex Transm Dis 1994;21:43-6.

16. Hammerschlag MR, Golden NH, Oh MK, et al. Single dose of azithromycin for the treatment of genital chlamydial infections in adolescents. J Pediatr 1993:122:961-5.

17. Lister PJ, Balechandran T, Ridgway GL, Robinson AJ. Comparison of azithromycin and doxycycline in the treatment of non-gonococcal urethritis in men. J Antimicrob Chemother 1993;31 (Suppl E): 185-92.

18. Lauharanta J, Saarinen K, Mustonen MT, Happonen HP. Single-dose oral azithromycin versus seven-day doxycycline in the treatment of non-gonococcal urethritis in males. J Antimicrob Chemother 1993;31 (Suppl E): 177-83.

19. Katz BP, Zwicki BW, Caine VA, Jones RB. Compliance with antibiotic therapy for Chlamydia trachomatis and Neisseria gonorrhoeae. Sex Transm Dis 1992;19:351-4

20. Grob PR. Antibiotic prescribing practices and patient compliance in the community. Scand $J$ Infect Dis 1992;(Suppl 83):7-14.

21. Genç M, Ruusuvaara L, Mardh P-A. An economic evaluation of screening for Chlamydia trachomatis in adolescent males. JAMA 1993;270:2057-64. 


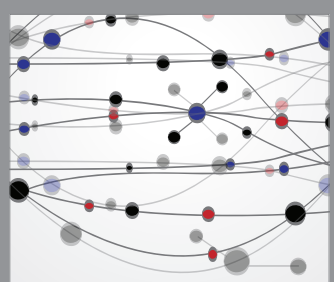

The Scientific World Journal
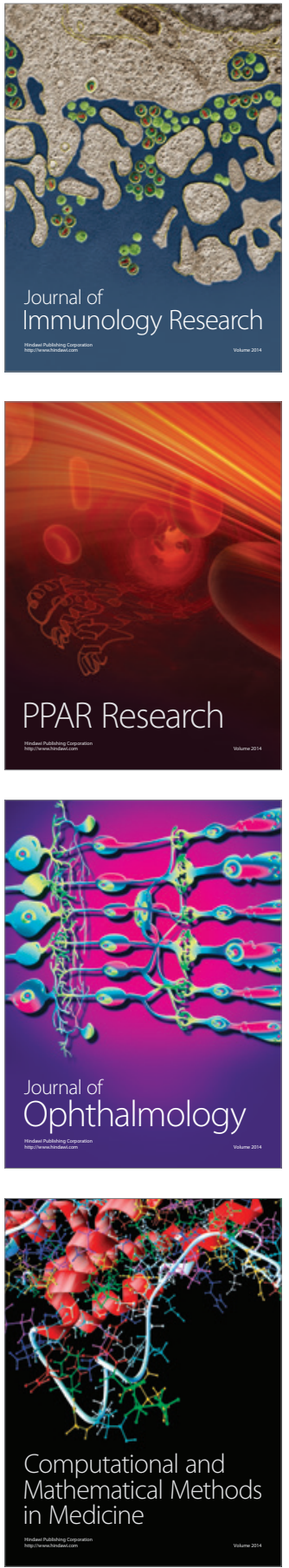

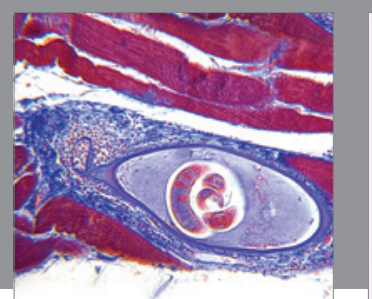

Gastroenterology Research and Practice

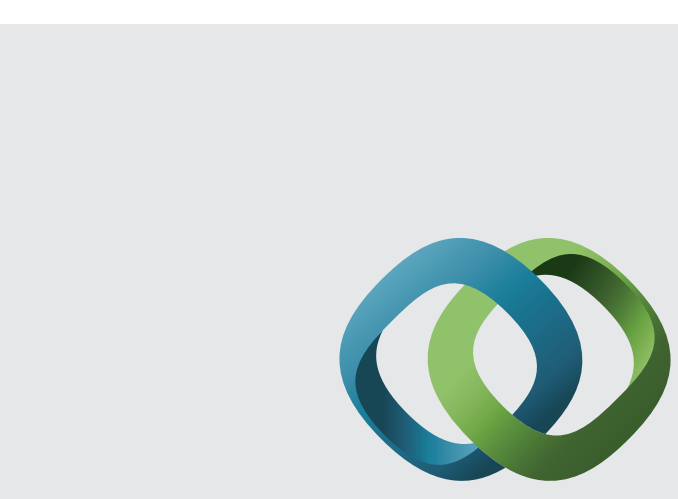

\section{Hindawi}

Submit your manuscripts at

http://www.hindawi.com
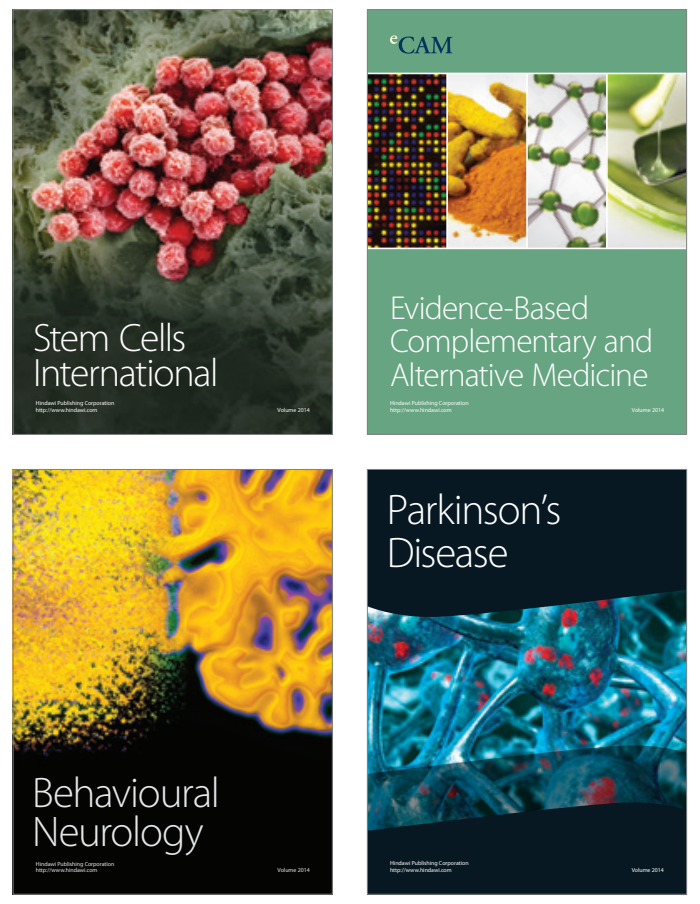
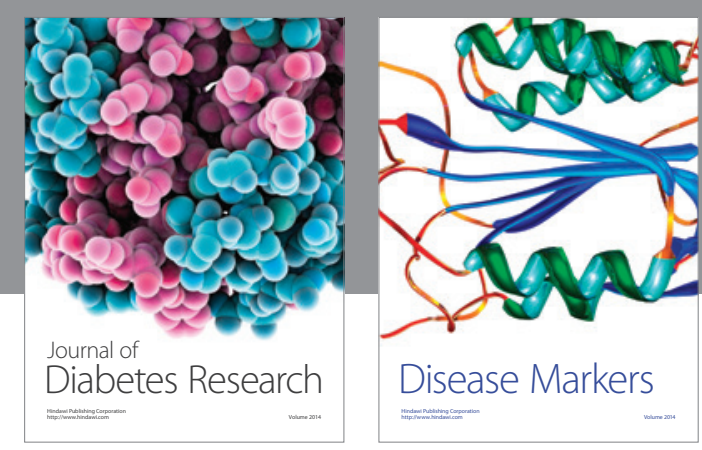

Disease Markers
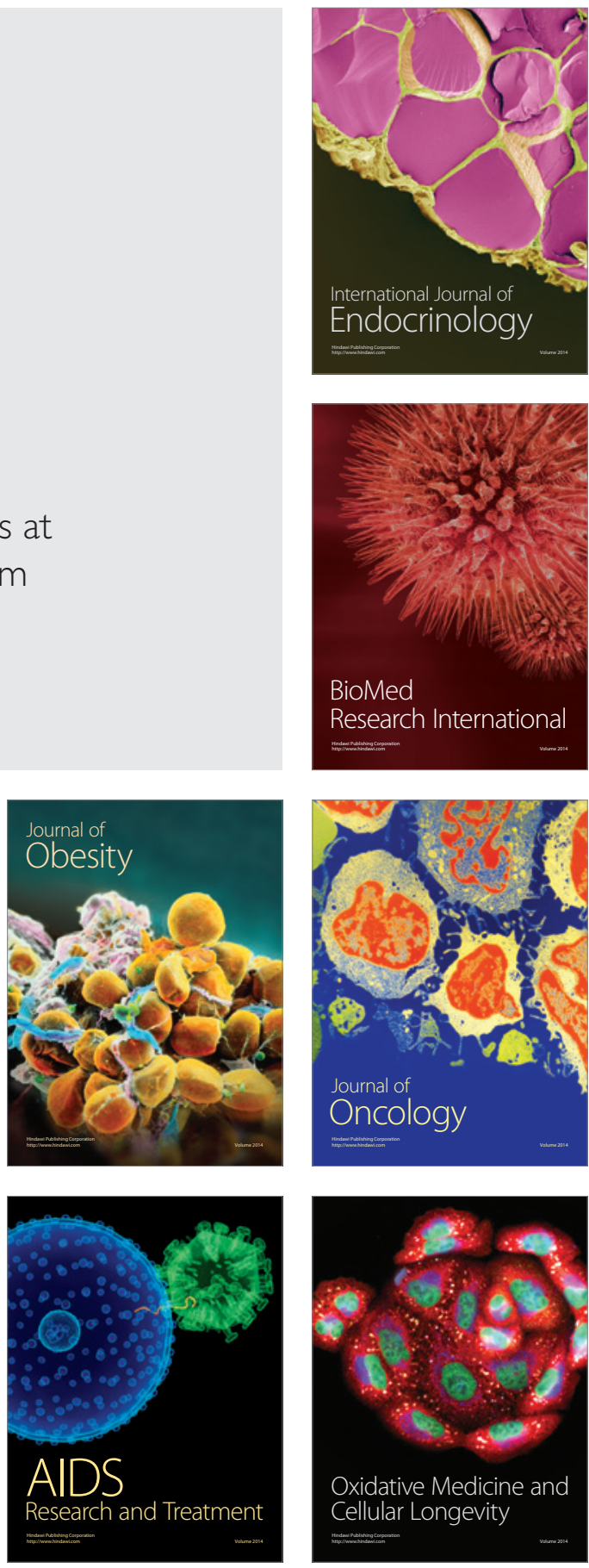